\title{
Observations on Multiplicative Composition in the Analytic Hierarchy Process
}

\author{
Thomas L. Saaty \\ University of Pittsburgh
}

\section{Introduction}

From the early years of development of the Analytic Hierarchy Process (AHP) I examined a number of alternatives for hierarchic composition that produced ratio scales. Ratio scales were always in my thinking because of my strong interest in physics. There one multiplies different ratio scales before and after raising them to powers. But even in mathematics, length, area and volume involve different powers of a metric. In my first book on the AHP I pointed out what is commonly known, that the product and quotient of different ratio scales is a ratio scale but not the sum or difference. The sum of elements from the same ratio scale is in fact a ratio scale. I also gave a theorem on the powers of a ratio scale which as products of ratio scales define a ratio scale. Later I worked out examples using multiplicative composition where to obtain the composite weight of an alternative, the weight of that alternative is raised to the power of its criterion and the product is taken over the criteria. In all the examples the outcome was close to that obtained by additive composition which is simply multiplying the weight of an alternative by that of its corresponding criterion and adding over the criteria. In this note I want to give several reasons for not using the multiplicative approach for hierarchic composition.

\section{Problems With Multiplicative Composition}

The AHP is a methodology that derives ratio scales from people's judgments. These judgments are intrinsically inconsistent. It is a good thing that we can allow for inconsistency in judgments for then they can be incrementally modified to allow for the effect of new information. Judgments are captured through paired comparisons expressing the dominance: importance, preference, or likelihood of one element over another. If the matrix is consistent, one can derive the rank of the elements by many different ways which all lead to the same 
outcome. One of them is based on multiplying the elements. However, another is based on adding the elements. When we admit inconsistency to capture numerical dominance, we need to both add and multiply the numerical values to capture the dominance of one element over another $B$ either directly or through intermediate paths of a pair of judgments, triple judgments, and so on at infinitum. Such transitivity is obtained by raising the matrix of judgments to all order powers and combining the results which yield the principal right eigenvector of the matrix of comparisons for the ranking weights. Thus when inconsistency is allowed, the eigenvector is the unique way to obtain the rank. It is obtained by both multiplying and adding judgments. To avoid the addition operation some people have felt justified demand that the judgments always be consistent, more as wishful thinking than a valid observation. The following example shows that with inconsistency, multiplicative composition leads to different (and wrong) results for the values and ranks of the alternatives.

Consider the inconsistent matrix

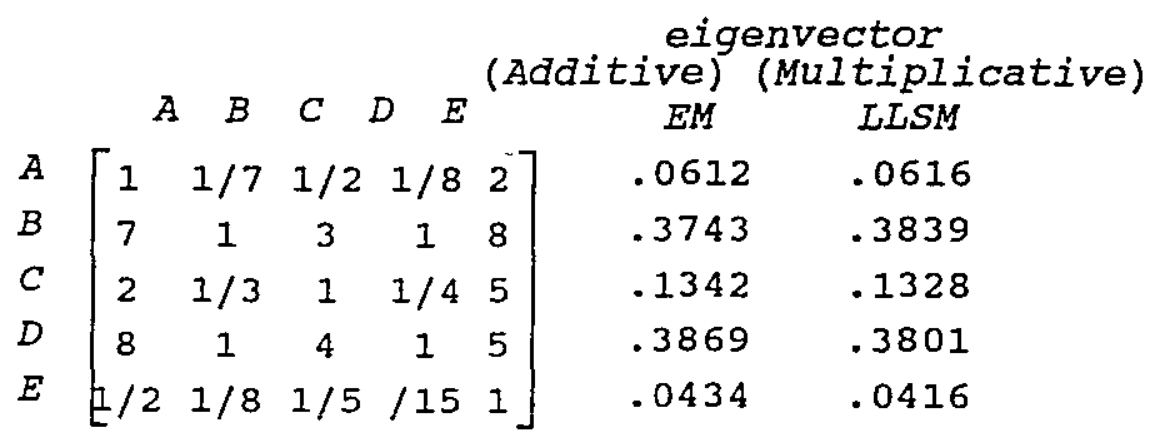

where $\mu(n)=.045$ and $\mu(n)[$ Random $\mu(n)]=.040$

The rankings are

$$
\begin{gathered}
w_{D}>w_{B}>w_{C}>w_{A}>w_{E} \\
u_{B}>u_{D}>u_{C}>u_{A}>u_{E}
\end{gathered}
$$

Again we note the counterintuitive outcome that if some element in an inhomogeneous set has a low priority relative to others, raising this priority to the power of its criterion (which is less than one) can make its global priority larger than its local priority. For example, if the local priority is 0.02 and the criterion priority is 0.50 , the global priority is $0.14147>0.02$. Also, if an element does not have a certain property and is directly assigned a zero value for that property, its multiplicative composition would net it a priority of zero on all the criteria. 
property, its multiplicative composition would net it a priority of zero on all the criteria.

Our third observation on not using multiplicative composition is derived from the analysis of dependence. The supermatrix is the AHP vehicle to represent dependence among the elements of a decision. Powers of the supermatrix capture the transitivity of dominance to obtain the rank along lines similar to those outlined above for the eigenvector. Additive hierarchic composition (not multiplicative composition) is a special case of the general approach of the supermatrix. In other words, additive composition is a corollary of the supermatrix approach.

Our fourth observation concerns rank. Claims have sometimes been made that multiplicative composition always preserves rank given as a reason for its adoption. But what does one do when rank should not be preserved? Is there yet another multiplicative method to allow rank to reverse? Real life examples have been given to show that a ranking method must preserve rank in some case and also allow rank to reverse in other cases. With additive composition in the AHP, the manner in which the weights of the alternatives are processed with respect to each criterion and then weighted and summed determines whether the outcome allows for rank reversal or preservation from irrelevant alternatives. In the distributive mode they are normalized and rank reversal can occur with respect to several criteria, in the ideal mode for each criterion the weights of the alternatives are divided by the largest value among them and rank is preserved.

\section{The Remainder Term - Multiplicative and Additive Give Close Results}

Our fifth reason for not using multiplicative composition is based on examining the closeness of ratios obtained from both additive and multiplicative composition. Let the relative weights of the criteria be $w_{1}, \ldots, w_{n}$ with $\sum_{j=1}^{n} w_{j}=1$.

Let $w_{i j}, \sum_{i=1}^{m} w_{i j}=1, j=, \ldots, n$ be the relative weight of alternative $i$ with respect to criterion $j$. Multiplicative composition yields $\prod_{j=1}^{n} w_{i j}^{w_{j}} \quad i=1, \ldots, m$ for the composite weight of alternative 
$i$ with respect to all the criteria. Now

$$
\log x=(x-1)-\frac{1}{2}(x-1)^{2}+\frac{1}{3}(x-1)^{3}-\cdots=(x-1)+R \quad 0<x<2 .
$$

where $R$ is the remainder. This series converges to $x$ in the indicated range and hence its remainder is bounded.

Theorem: $\prod_{j=1}^{n} w_{i j}^{w_{j}}=\sum_{j=1}^{n} w_{j} w_{i j}+R_{i} i=1, \ldots, m$

Recalling that the alternatives are few and homogenous so that none of them is zero (or near zero) we have

$$
\log \prod_{j=1}^{n} w_{i j}^{w_{j}}=\sum_{j=1}^{n} w_{j} \log w_{i j}=\sum_{j=1}^{n} w_{j}\left(w_{i j}-1\right)+Q_{i}=\left[\sum_{j=1}^{n} w_{j} w_{i j}-1+Q_{i}\right] \quad i=1, \ldots, m
$$

After taking the logarithm we need to take the exponential to restore the original expression. If we use $R_{i}$ to denote the remainder in our series expansion we have:

$$
\exp [\quad]=1+\sum_{j=1}^{n} w_{j} w_{i j}-1+R_{i} \quad i=1, \ldots, m
$$

where $R$ is the remainder.

Thus, to a first order approximation we have:

$$
\prod_{j=1}^{n} w_{i j}^{w_{j}}=\sum_{j=1}^{n} w_{j} w_{i j}+R_{i} \quad i=1, \ldots, m
$$

Because the first term on the right is normalized to unity when summed over $i$, we also normalize the left side so that

$$
\sum_{i=1}^{m} \prod_{j=1}^{n} w_{i j}^{w_{j}}=1
$$

and (1) becomes

$$
\prod_{j=1}^{n} w_{i j}^{w_{j}} / \sum_{i=1}^{m} \prod_{j=1}^{n} w_{i j}^{w_{j}}=\sum_{j=1}^{n} w_{j} w_{i}+R_{i} \quad i=1, \ldots, m
$$


The question raised by the above representation does not necessarily have to do with the size of the remainder. The representation in (2) suggests two ideas. The first is that the right side be written as a multiplicative perturbation of the left side. Thus instead of adding a remainder, we write

$$
\prod_{j=1}^{n} w_{i j}^{n} / \sum_{i=1}^{m} \prod_{j=1}^{n} w_{i j}^{w_{j}}=\left(\sum_{j=1}^{n} w_{j} w_{i j}\right) \varepsilon_{i}
$$

Where the perturbation $\varepsilon_{i}$ is positive. The other idea is that ratios formed by using the multiplicative term on the left of (3) may be close to ratios formed by the additive term in (3). To test the closeness or the compatibility of all such ratios we performed a random simulation exercise of $2500 \times 81$ experiments to determine the closeness of the two sides of (2) normalized. We systematically increased the number of criteria from 2 to 10 and the number of alternatives from 2 to 10 . For each number of criteria and of alternatives, we assigned the criteria and the alternatives random values from a uniform distribution on $(0,1]$ that were then normalized and combined by the two methods. For each number of criteria and alternatives 2500 experiments were performed. Each time absolute differences were taken between the two sides for each alternative and the results were analyzed for the alternatives. The outcomes were then averaged across all 2500 experiments to yield the mean difference.

We then used our ratio scale metric $\frac{1}{n^{2}} e^{T} A^{\circ} B^{T} e$ to evaluate the closeness of the same 2500 experiments for each number of alternatives and criteria. Here we formed a matrix $A$ of all possible ratios of the multiplicative ratio scale. Each row of $A$ gives the ratios of one element with respect to each other element. We did the same for the additive ratio scale to define a matrix $B$. We then formed a new matrix $A^{\circ} B^{T}$ (where $B^{T}$ is the transpose of $B$ ) each of whose entries is a ratio of corresponding entries of the first and of the second matrix respectively. If the scales are identical each entry of this matrix would be equal to one. Finally, we summed the elements of $A^{\circ} B^{T}$ which corresponds to performing the operation $e^{T} A^{\circ} B^{T} e$ where $e^{T}=(1,1, \ldots, 1)$. Since the sum of the elements of $A^{\circ} B^{T}$ is $\geq n^{2}$ where $n$ is the order of the matrix we divided by $n^{2}$ [1]. In general, when two scales are close we say that they are 
compatible if $e^{T} \frac{1}{n^{2}} A^{\circ} B^{T} e \leq 1.10$. The outcome of this exercise given in the table below shows that except for the case of two criteria and more than six alternatives, the two sides of (2) are close according to their mean differences and compatible according to their mean ratios. Statistical regression analysis shows that the mean difference values cannot be explained by the number of criteria and of alternatives. However, the mean metric values could be explained with greater confidence by using both these parameters.

\section{Conclusions}

Our experiments with the exception of a part of the two criteria case, show that multiplicative composition does not add significant information to the ratios obtained from additive composition. Because the ratio scales used on both sides of (3) give close results one may conclude that the two methods can be used interchangeably but that is not true. We have seen that if the judgments are inconsistent one must use the principal eigenvector and additive composition and not the multiplicative approach to capture the right priorities and ranks. This approach is also true of the supermatrix from which we deduce additive hierarchic composition as a corollary.

\section{References}

1. Saaty, T.L., 1994, "A Ratio Scale Metric and the Compatibility of Ratio Scales: On the Possibility of Arrow's Impossibility Theorem", Applied Mathematics Letters (to appear). 


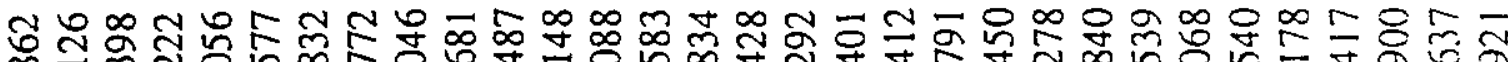

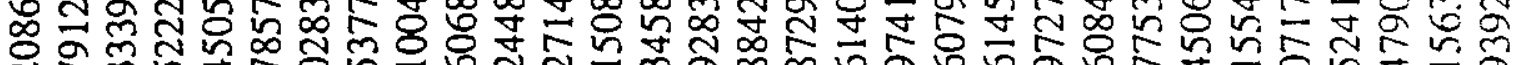

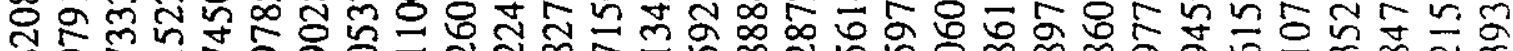

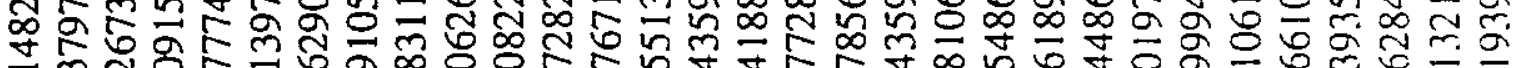

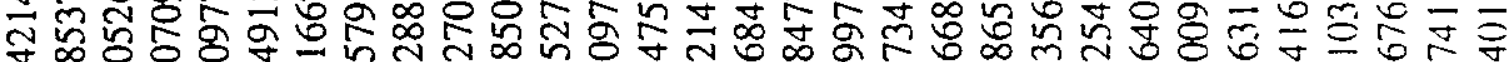

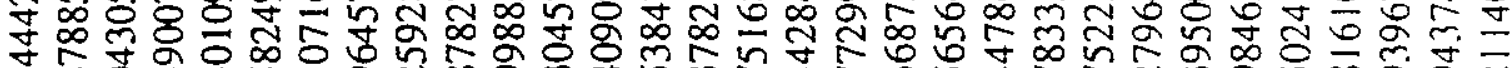

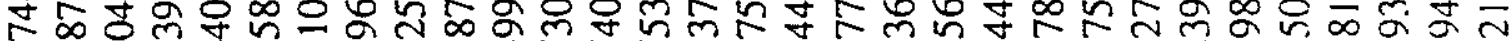

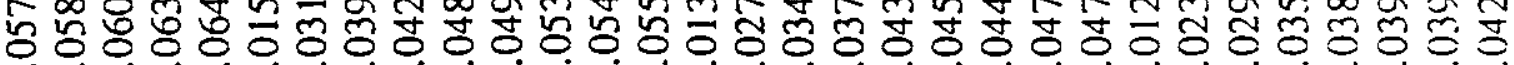

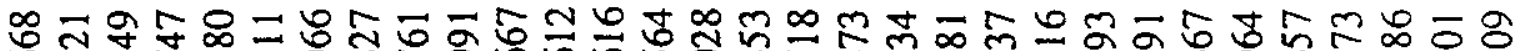

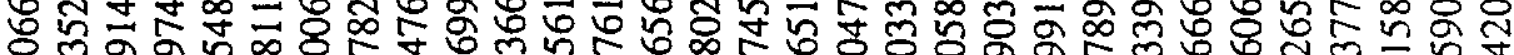

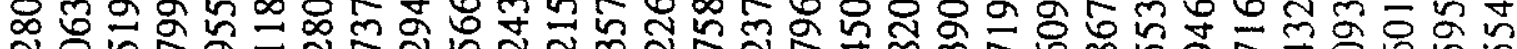

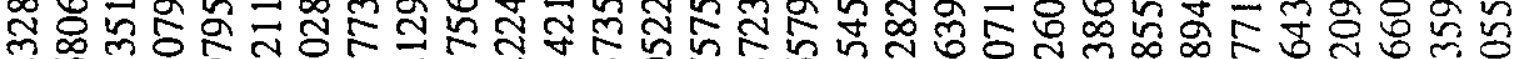

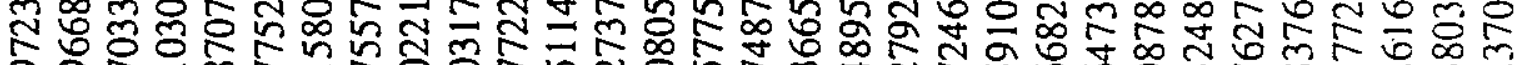

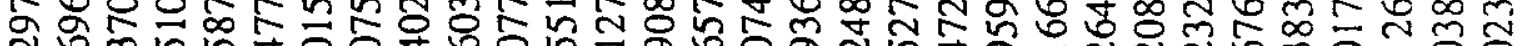
거의

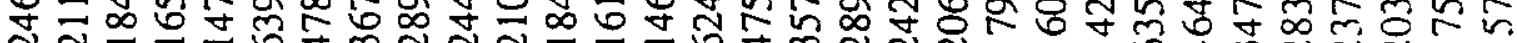

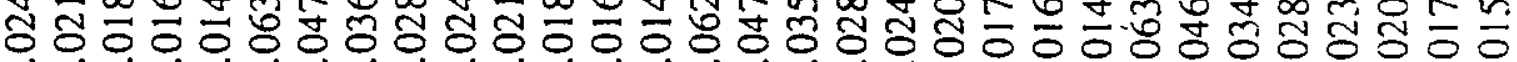

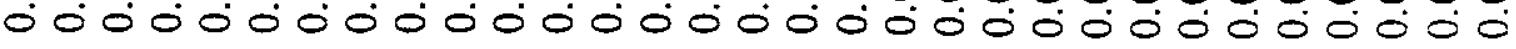

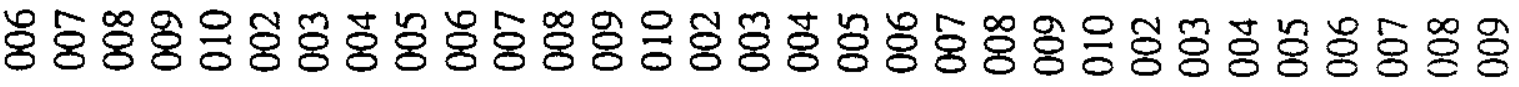

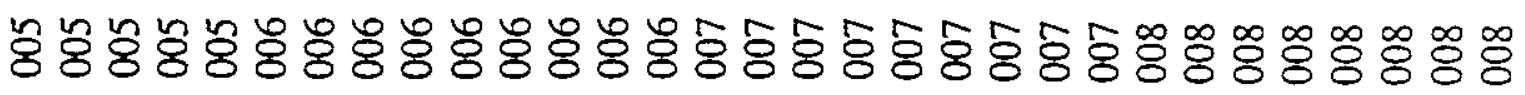

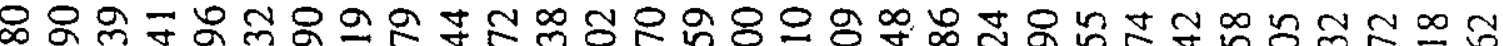
オ

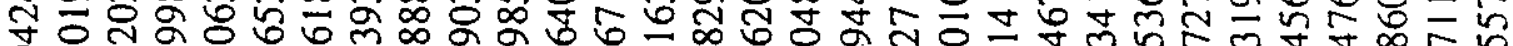
O이에 "ె5

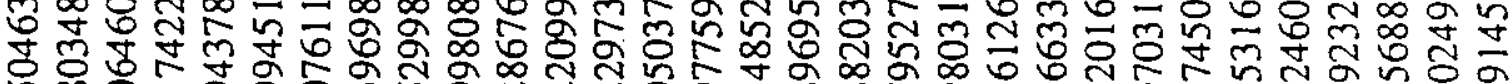
舟

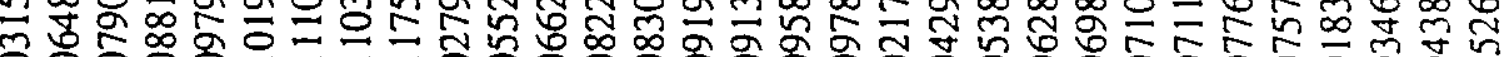
\%

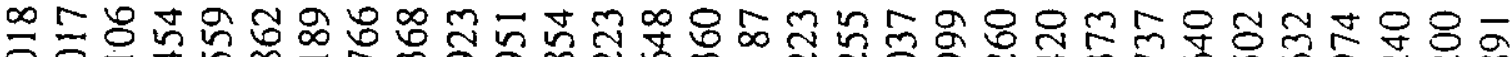
б

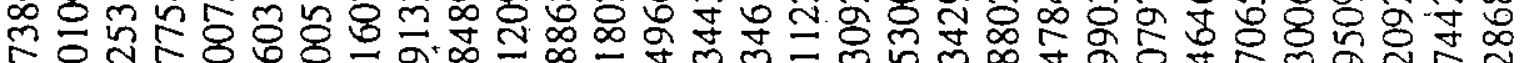

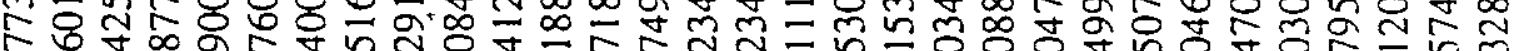

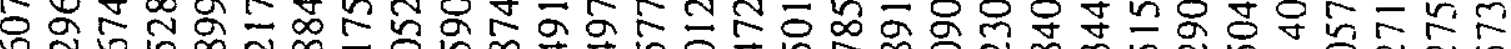

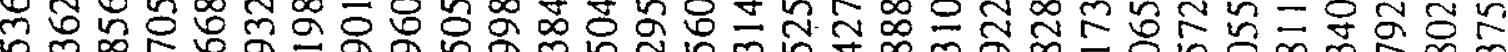
n

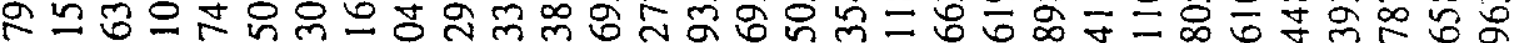
๙ิ

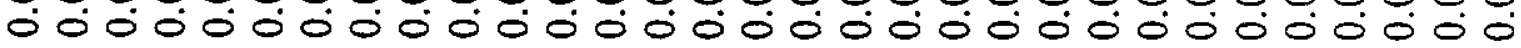

啳

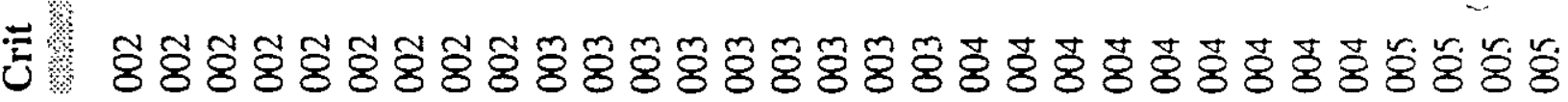




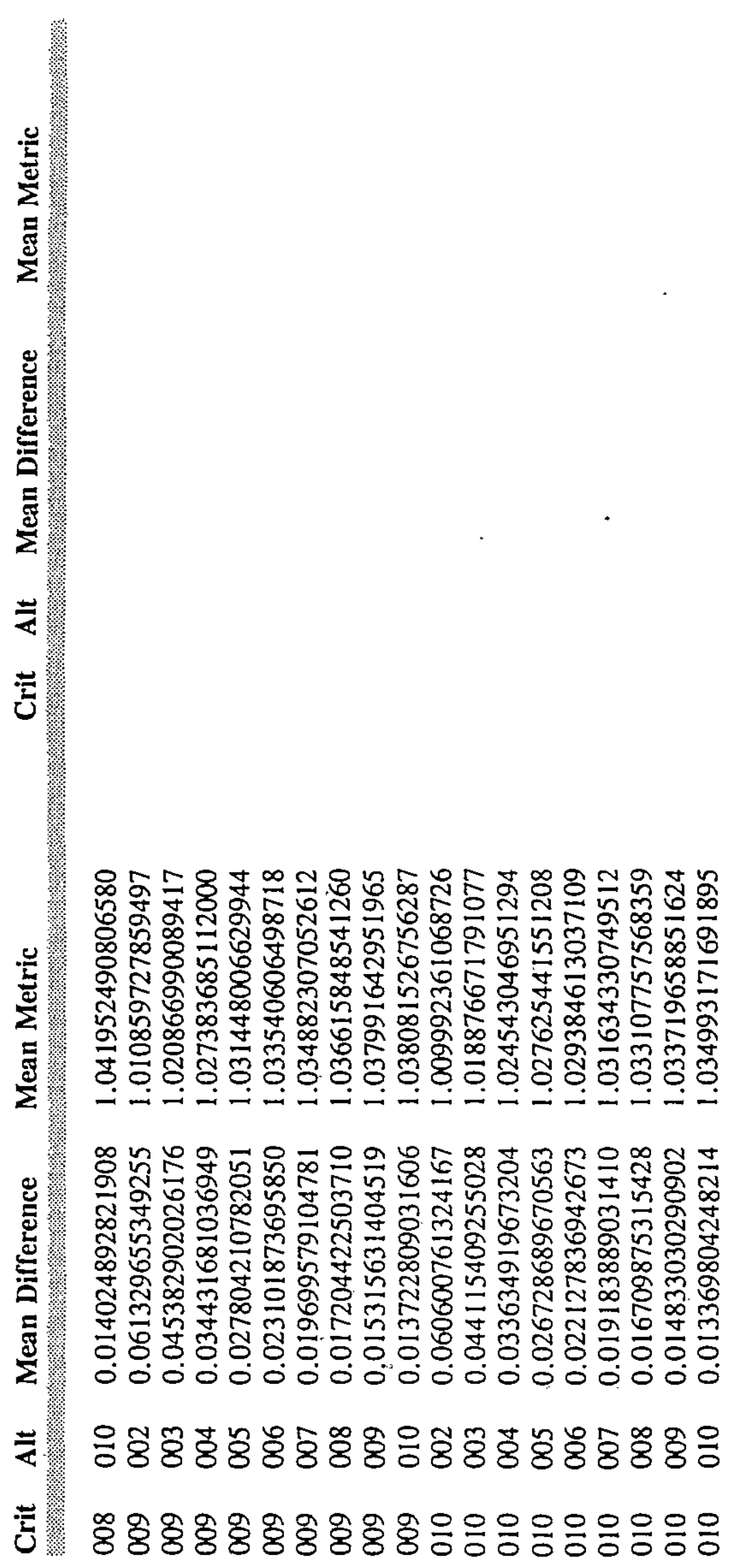

